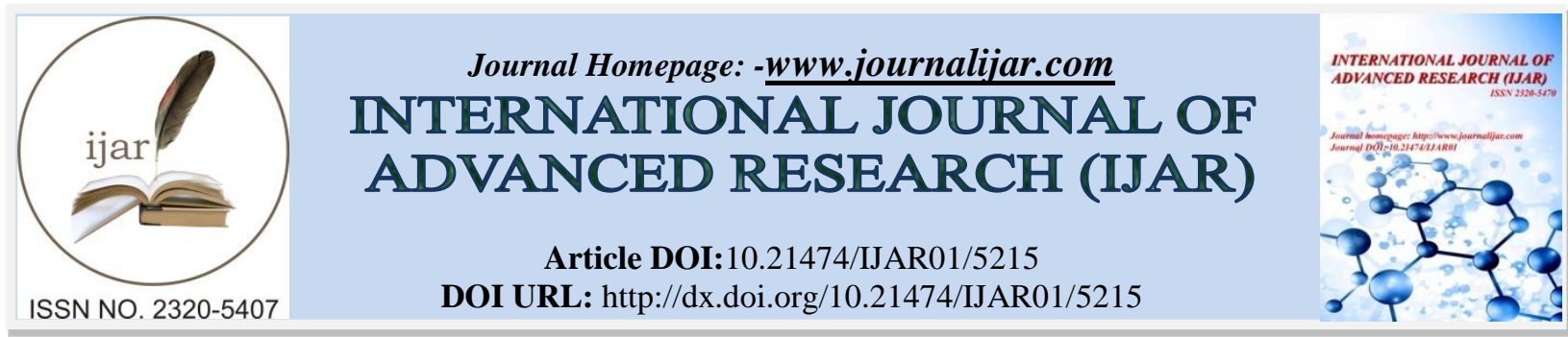

RESEARCH ARTICLE

\title{
IMMUNOHISTOCHEMICAL EXPRESSION OF CYCLIN D1 AND KI 67 IN PREMALIGNANT AND MALIGNANT PROSTATIC LESIONS.
}

Samia M. Gabal MD ${ }^{1}$, Samar A. El Sheikh M.D ${ }^{1}$, Hala M. El-hanbuli MD ${ }^{2}$, Maram M. Ayoub, M.B. B.Ch² Department of Pathology, Faculty of Medicine, Cairo University ${ }^{1}$ and Fayoum University ${ }^{2}$, Egypt.

\section{Manuscript Info}

Manuscript History

Received: 19 June 2017

Final Accepted: 21 July 2017

Published: August 2017

Keywords:-

Cyclin D1, Ki-67, Prostatic

adenocarcinoma.

\begin{abstract}
Background: Prostatic carcinoma is a common and growing public health problem. Cyclin D1 is an amino acid that is expressed in the cell cycle and has an important role in regulating cancer progression, also, The Ki-67 protein functions as a nuclear antigen that is only expressed in proliferating cells. It is a marker of the growth fraction in malignant tissue.

Objectives: to study the expression of Cyclin D1 and Ki-67 in prostatic adenocarcinoma and PIN and correlate their expression with age, preoperative serum PSA and Gleason score.

Material and Methods: A total of 50 cases including 25 cases of prostatic carcinoma, 10 cases of high grade PIN and 15cases of BPH (as a control group) were studied. Tumor grade was determined according to Gleason's grading system. Cyclin D1 and Ki-67 expression was determined by IHC staining.

Results: In carcinoma cases, Cyclin D1 was expressed in 23/25 cases(92\%), in PIN it was expressed in $8 / 10$ cases (80\%) while in BPH it was expressed in only $3 / 15$ cases(20\%). Ki-67 was expressed in 20 / 25 (80\%) cancer cases, in PIN it was expressed in 7 / 10 (70\%) cases while in BPH it was expressed in only $1 / 15$ case $(6.7 \%)$. Both Cyclin D1 and Ki-67 were significantly up-regulated in cancer cases as compared to BPH cases $(\mathrm{P}<0.001)$. No significant correlation was found between the intensity of Cyclin D1 expression and Gleason score, whereas a statistically significant correlation was observed between the intensity of Ki 67 expression and Gleason score (P < 0.001). A statistically significant positive correlation was observed between Cyclin D1 and Ki-67 expression in cancer group, PIN group and $\mathrm{BPH}$ group with $\mathrm{P}$ value $0.03,0.001$ and 0.04 respectively.

Conclusion: Cyclin D1 may be helpful in differentiation between BPH, PIN and adenocarcinoma of the prostate, while Ki-67 can be applied along with other prostate cancer prognostic factors.
\end{abstract}

Copy Right, IJAR, 2017,. All rights reserved. 


\section{Introduction:-}

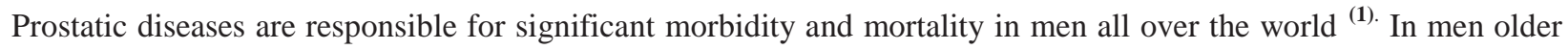
than 50 years, prostatic carcinoma represents the most common malignant tumor and is characterized by a highly variable clinical outcome ${ }^{(\mathbf{2})}$. It accounts for about $50 \%$ of male genital tract malignancies in Egypt ${ }^{(3)}$, and prostatic intraepithelial neoplasia (PIN), is probably the most important precursor lesion of invasive prostatic cancer ${ }^{\text {(4). }}$

Cyclin D1 has been identified as a short-lived nuclear protein involved in cell cycle transition from growth (G1) to synthesis (S) phase in both normal and neoplastic cells ${ }^{(5)}$.The relationship between cyclin D1 expression and prostate cancer remains controversial. Some researchers have found occasional cyclin D1 expression in prostate cancer, whereas others reported that prostatic tumors with high cyclin D1 expression were associated with a more aggressive disease ${ }^{(6)}$

$\mathrm{Ki}-67$ is a protein involved in cell cycle regulation and cell proliferation. It is expressed in proliferating cells during all active phases of the cell cycle (apart from G0). It is confirmed to be the most promising biomarker for routine practice applications ${ }^{(7),}$ which may reflect the biologically malignant potential of prostatic carcinoma and justify its use in selecting candidates for active surveillance However, its significance remains controversial ${ }^{(\mathbf{8})}$

\section{Aim of the work:-}

Because the role of cyclin D1 and Ki-67 in prostate cancer is unclear, we studied the expression of cyclin D1 and $\mathrm{Ki}-67$ in PIN, prostatic adenocarcinoma and benign prostatic hyperplasia (BPH) (as a control group) to evaluate the relationship of these proteins with some clinicopathological features as age, preoperative serum PSA and Gleason score and to declare a possible correlation between Cyclin D1 and Ki-67.

\section{Material and Methods:-}

\section{Specimens:-}

Paraffin blocks prepared from 50 specimens of prostatic tissue were collected in this study, including: 7 radical prostatectomy specimens, 18 transurethral specimens (TUR), 22 transrectal needle biopsies (TRUS) and 3 cases of open prostatectomy. The specimens were obtained from the Department of Pathology, Faculty of Medicine, Cairo University, in the period from January 2013 to December 2014. The specimens included 25 cases of previously diagnosed prostatic carcinoma, 10 cases of high grade PIN and 15 cases of BPH (as a control group). For all cases, available clinical data such as age of the patients and preoperative serum Prostate-specific antigen (PSA) were evaluated.

\section{Histopathological examination:-}

Each paraffin block was recut into serial sections and stained by Hematoxylin and Eosin (H\&E). In histopathological evaluation of the prostatic adenocarcinoma, Gleason grading system was used modified by Amin et al. ${ }^{(9)}$.

\section{Immunohistochemical staining:-}

Immunohistochemical analysis was performed on routinely processed, formalin-fixed, paraffin-embedded tissue. Tissue sections were cut at 5 microns thickness, mounted on charged slides and stained by Cyclin D1 using Monoclonal Mouse anti-human Cyclin D1 (SPM587-NBP2-32840,Novus Biological, USA) and by Ki-67 using polyclonal Rabbit anti-human Ki-67(NB500-170, Novus Biological, USA).

After routine deparafinization in xylene, the sections were hydrated through a series of graded alcohols, distilled water, and phosphate-buffered saline (PBS) at ph.7.2-7.4.Antigen retrieval was performed using Tris-EDTA (PH.9) at the microwave for Cyclin D1 and using citrate buffer $(\mathrm{PH}=6)$ for Ki67 immunostaining.

Blocking reagent (Ultra V Block) was placed on each slide and incubated for 10 minutes at room temperature in the humidity chamber. Excess serum was shacked off without washing. Each primary antibody was placed on the slides. The slides were incubated overnight at room temperature in humid chamber. The slides were rinsed for 5 minutes. 2-3 drops of secondary antibody (biotinylated polyvalent) diluted in this buffer solution TBS, were placed on each slide. The slides were incubated in a humidity chamber at room temperature for 15 minutes. After tapping off excess buffer the slides were rinsed for 5 minutes, twice, in PBS and were incubated in Streptavidin enzyme for 15 minutes at room temperature. The slides then rinsed for 5 minutes, twice, in PBS. Working Diaminoben zidine tetrhychochloride (DAB) reagent was placed on each slide (approximately $500 \mathrm{ml}$ per section). The slides were incubated for 4 minutes at room temperature in the humidity chamber and then washed thoroughly in distilled water. Counter stain with Mayer's hematoxyline for 1 minute was done then the slides were washed. The slides were placed 
in two changes of $95 \%$ ethyl alcohol, then two changes of absolute alcohol, each for two minutes. Finally, the slides were cleared in xylene and cover slips were fixed using mounting reagent. Each slide was evaluated by the authors.

\section{Evaluation of Cyclin D1 expression:}

Cyclin D1 expression was graded on the basis of the intensity of staining within the tumor cells. Only nuclear staining was considered positive, while isolated cytoplasmic staining whether in tumor tissue or in few foci of benign prostatic tissue found adjacent to tumor was ignored ${ }^{(\mathbf{1 0})}$.

The scoring system used for evaluation of Cyclin D1 expression ${ }^{(11)}$ was as follows:

1. Negative (no staining)

2. Mild (nuclear staining of $<10 \%$ cells),

3. Moderate (staining of $10-49 \%$ cells)

4. Strong (staining of $\geq 50 \%$ cells).

\section{Evaluation of Ki 67 expression:}

The tumors were divided into five groups regarding the percentage of Ki-67 nuclear stained cells ${ }^{(12)}$.

1. Cases in which the percentage of stained cells was $\leq 2 \%$ were considered negative.

2. Cases with Ki-67 index of $>2 \%-\leq 25 \%$ were considered $1+$

3. $26-50 \%$ as $2+$

4. $51-75 \%$ as $3+$

5. $76-100 \%$ as $4+$

\section{Statistical analysis:-}

SPSS (statistical package for social sciences) version 22.0 was used for data management and data analysis. Mean Istandard deviation described quantitative variables and median with range when appropriate (distribution did not follow normality). Number and percentages described qualitative data and Chi-square or / Fisher exact tested proportion independence. For comparing mean values of 2 independent groups, parametric and non-parametric t test were used. For comparing means of more than two independent groups one way ANOVA (analysis of variance) and Kruskal Wallis ANOVA were used. Correlation analysis was used to show strength and significance of association between quantitative variables. $\mathrm{P}$ value is always 2 tailed and significant at 0.05 level.

\section{Results:-}

Among the fifty studied cases, twenty five (50\%) were previously diagnosed as prostatic adenocarcinoma, fifteen cases $(30 \%)$ were diagnosed as BPH and ten (20\%) cases were diagnosed as PIN based on hematoxylin and eosinstained sections.

The age of the studied cases was between 50 -85 years old and the highest median was 67 which was detected in prostatic carcinoma group. The preoperative serum PSA in the studied cases ranged from $1 \mathrm{ng} / \mathrm{dl} \mathrm{up}$ to $313 \mathrm{ng} / \mathrm{dl}$ with a highest PSA mean value seen in prostatic carcinoma group (Table 1).

Table(1):-Comparison of age and pre-operative PSA level in the studied groups.

\begin{tabular}{|c|l|c|c|c|}
\hline \multicolumn{2}{|c|}{ Variable } & $\begin{array}{c}\text { Prostatic cancer } \\
\text { N=25 }\end{array}$ & BPH \\
\hline \multirow{3}{*}{ Age } & Minimum & N=15 & N=10 \\
\cline { 2 - 5 } & Maximum & 55 & 50 & 50 \\
\cline { 2 - 5 } & Mean & 83 & 85 & 80 \\
\hline \multirow{3}{*}{ PSA } & Minimum & 67 & 65 & 3 \\
\cline { 2 - 6 } & Maximum & 313 & 26 & 32 \\
\cline { 2 - 6 } & Mean & 56.43 & 6.07 & 12.32 \\
\hline
\end{tabular}


The Gleason scoring of the studied prostatic carcinoma cases ranged between $4-10$. The most prevalent score in the studied cases was Gleason score 8 (28\%) (Table 2).

Table (2):-Distribution of prostatic carcinoma cases according to Gleason score

\begin{tabular}{|c|c|c|}
\hline Gleason Score & Number of cases & Percentage (\%) \\
\hline Score 4 & 1 & $4 \%$ \\
\hline Score 5 & 1 & $4 \%$ \\
\hline Score 6 & 5 & $20 \%$ \\
\hline Score 7 & 6 & $24 \%$ \\
\hline Score 8 & 7 & $28 \%$ \\
\hline Score 9 & 4 & $16 \%$ \\
\hline Score 10 & 1 & $4 \%$ \\
\hline Total & $\mathbf{2 5}$ & $\mathbf{1 0 0 \%}$ \\
\hline
\end{tabular}

Regarding Cyclin D1 immunohistochemical staining (Figures 1, 2); there was a statistically significant difference in expression of cyclin D1 between BPH compared with PIN and carcinoma cases $(\mathrm{P}<0.001)$ with the highest expression in carcinoma cases (Table 3).

Table (3):- Cyclin D1 expression in all studied cases.

\begin{tabular}{|l|c|c|c|}
\hline \multicolumn{1}{|c|}{ Cyclin D1 scoring } & $\begin{array}{c}\text { Prostatic cancer cases } \\
\mathbf{N = 2 5 ( 1 0 0 \% )}\end{array}$ & $\begin{array}{c}\text { PIN } \\
\mathbf{N = 1 0}(\mathbf{1 0 0 \%})\end{array}$ & $\begin{array}{c}\text { BPH } \\
\mathbf{N = 1 5}(\mathbf{1 0 0 \%})\end{array}$ \\
\hline Negative & $2(8)$ & $2(20)$ & $12(80)$ \\
\hline Mild & $5(20)$ & $4(40)$ & $3(20)$ \\
\hline Moderate & $10(40)$ & $2(20)$ & $0(0)$ \\
\hline Strong & $8(32)$ & $2(20)$ & $0(0)$ \\
\hline
\end{tabular}

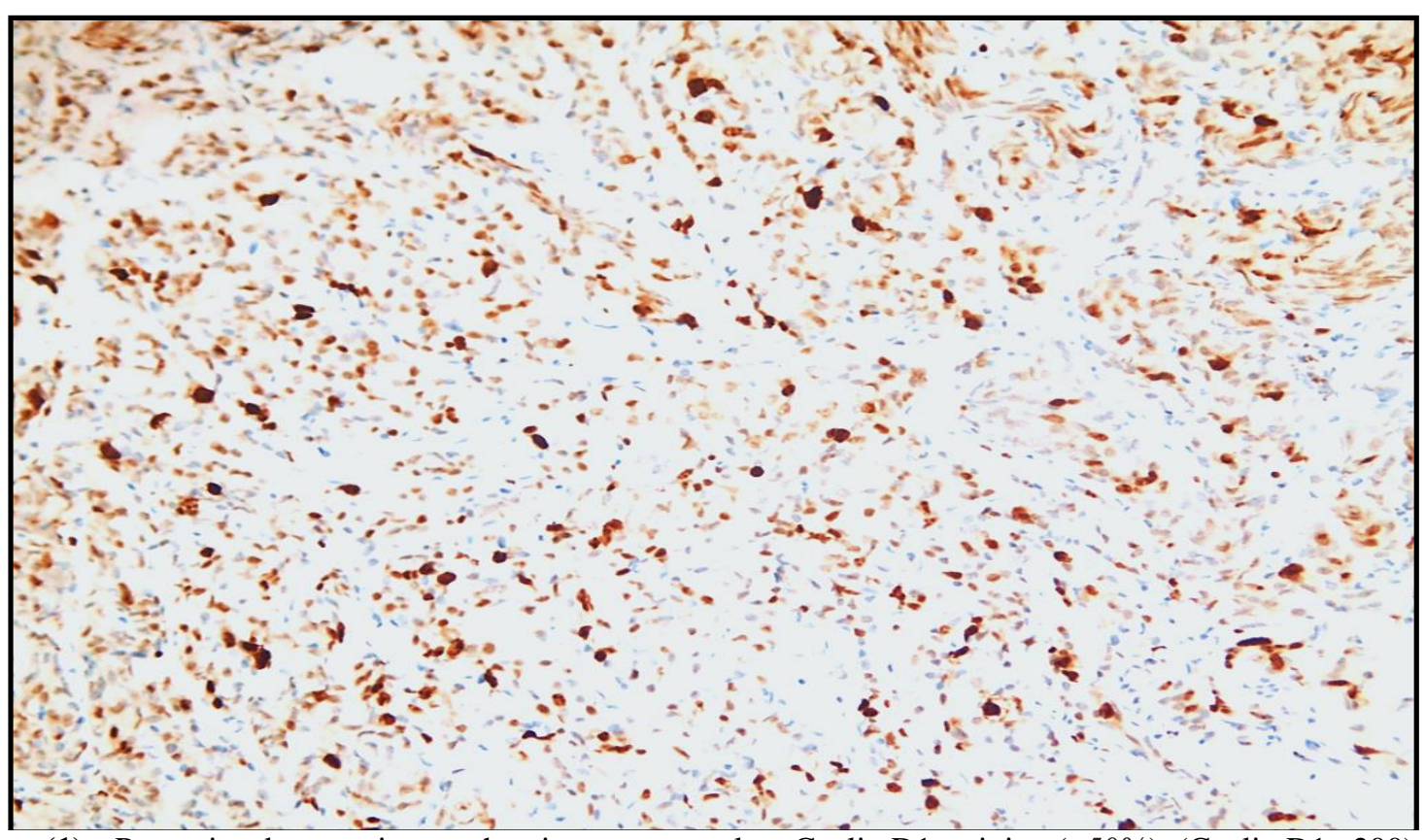

Figure (1):- Prostatic adenocarcinoma showing strong nuclear Cyclin D1 staining ( $\geq 50 \%)$. (Cyclin D1 x200) 


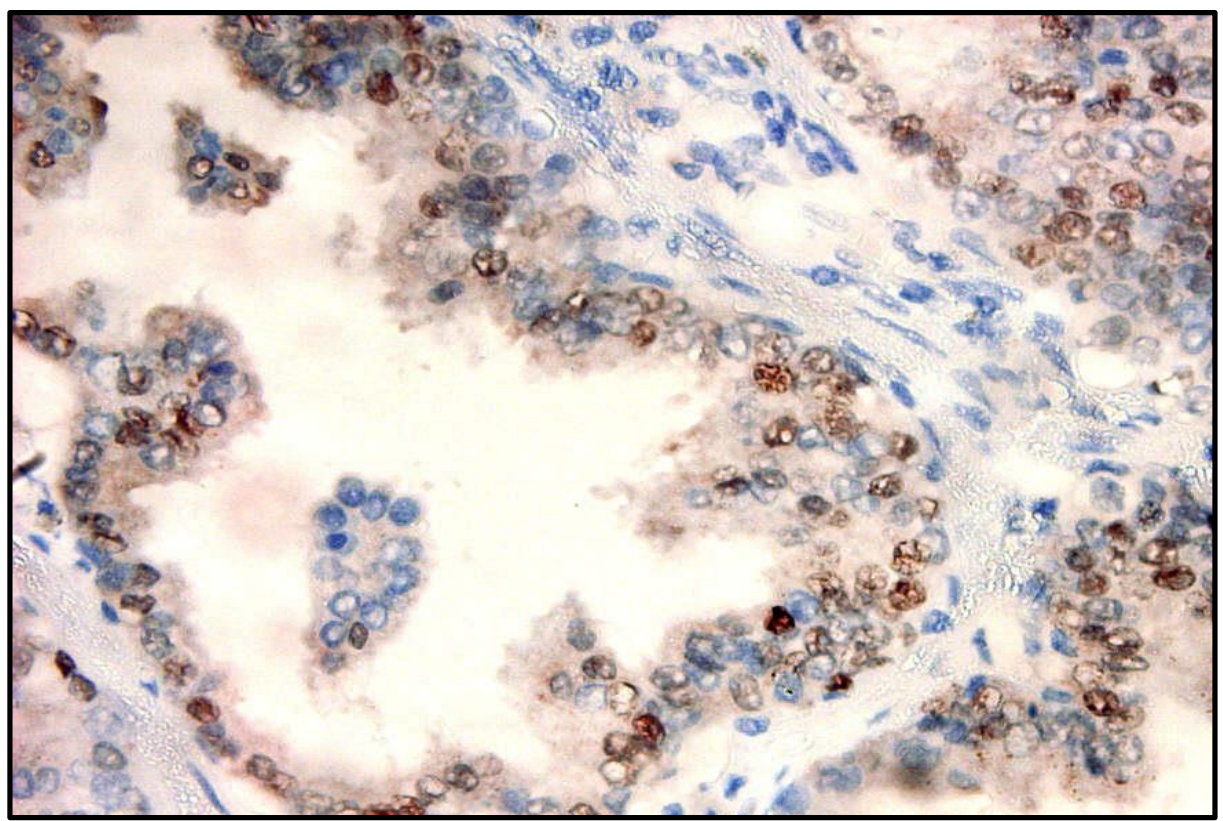

Figure (2):- High grade prostatic intraepithelial neoplasia showing positive Cyclin D1 staining. (Cyclin D1 $\mathrm{x} 400)$.

No significant relationship was found between the intensity of Cyclin D1 expression in prostate cancer cases and other clinicopathological factors as age of the patient $(\mathrm{P}=0.687)$ and preoperative PSA $(\mathrm{P}=0.286)$. Also there is no significant association or correlation was found between the intensity of Cyclin D1 expression and Gleason scoring of cancer prostate cases $(\mathrm{P}=0.337)$ (Table 4).

Table (4):- Correlation between Cyclin D1 expression in prostatic carcinoma cases and age, PSA and Gleason score:

\begin{tabular}{|ll|c|c|c|}
\hline & Age & PSA & Gleason score \\
\hline Cyclin D1 & Pearson Correlation & 0.085 & 0.222 & 0.200 \\
P value & 0.687 & 0.286 & 0.337 \\
\hline
\end{tabular}

Regarding Ki67 immunohistochemical staining (Figures 3,4); there was a statistically significant difference in expression of Ki-67 between BPH and prostate carcinoma is observed ( $\mathrm{P}$ value of < 0.001) (Table 5).

Table (5):- Ki-67 expression in all studied groups.

\begin{tabular}{|c|c|c|c|}
\hline Ki-67 scoring & $\begin{array}{c}\text { Prostatic cancer cases } \\
\mathrm{N}=\mathbf{2 5}(\mathbf{1 0 0 \%})\end{array}$ & $\begin{array}{c}\text { PIN cases } \\
\mathrm{N}=10(100 \%)\end{array}$ & $\begin{array}{c}\text { BPH cases } \\
\mathrm{N}=15(100 \%)\end{array}$ \\
\hline Negative $(\leq 2 \%$ ) & $5(20)$ & $3(30)$ & $14(93.3)$ \\
\hline$+1(>2 \%-\leq 25 \%)$ & $1(4)$ & $1(10)$ & $1(6.7)$ \\
\hline$+2(26-50 \%)$ & $7(28)$ & $3(30)$ & $0(0)$ \\
\hline$+3(51-75 \%)$ & $8(32)$ & $3(30)$ & $0(0)$ \\
\hline$+4(76-100 \%)$ & $4(16)$ & $0(0)$ & $0(0)$ \\
\hline
\end{tabular}




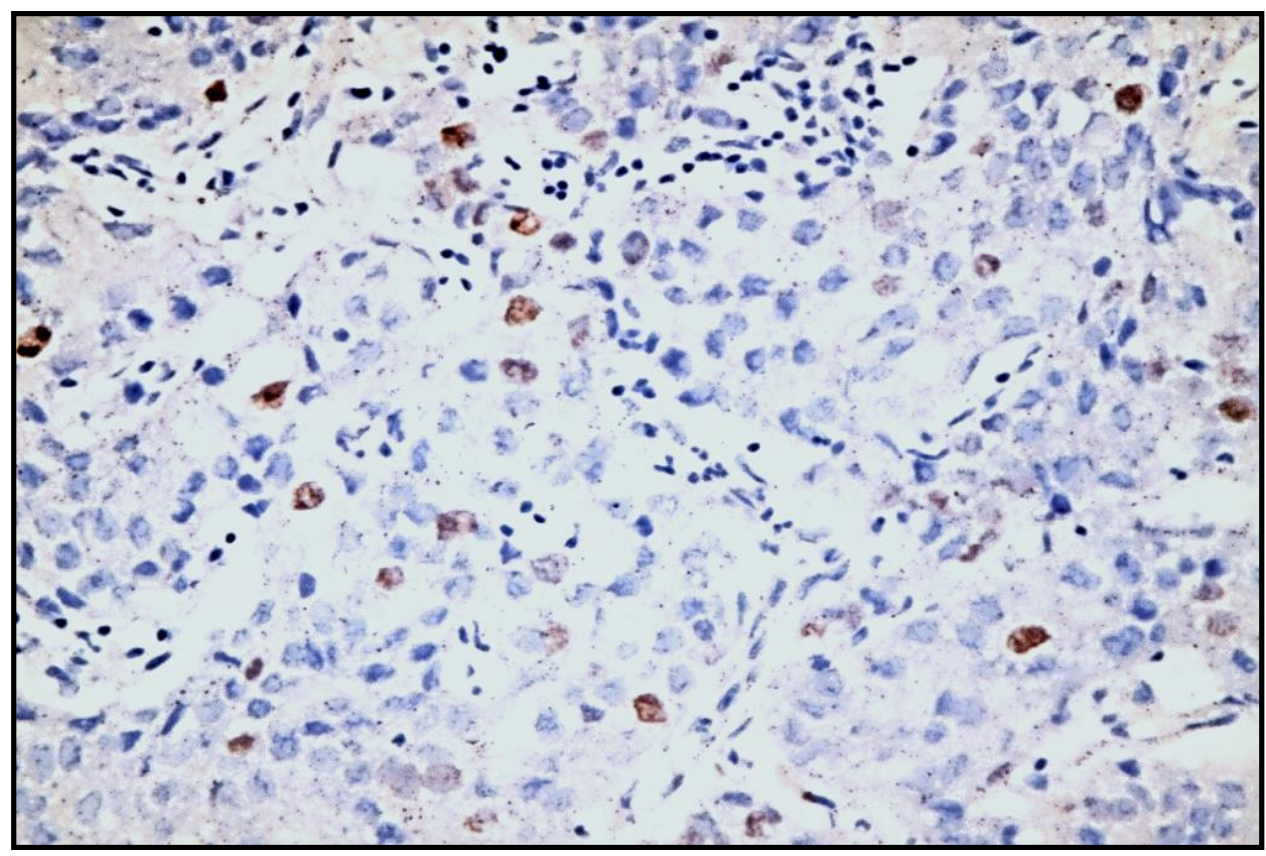

Figure (3):- Prostatic adenocarcinoma showing 2+ nuclear Ki 67 staining. (Ki67 x200).

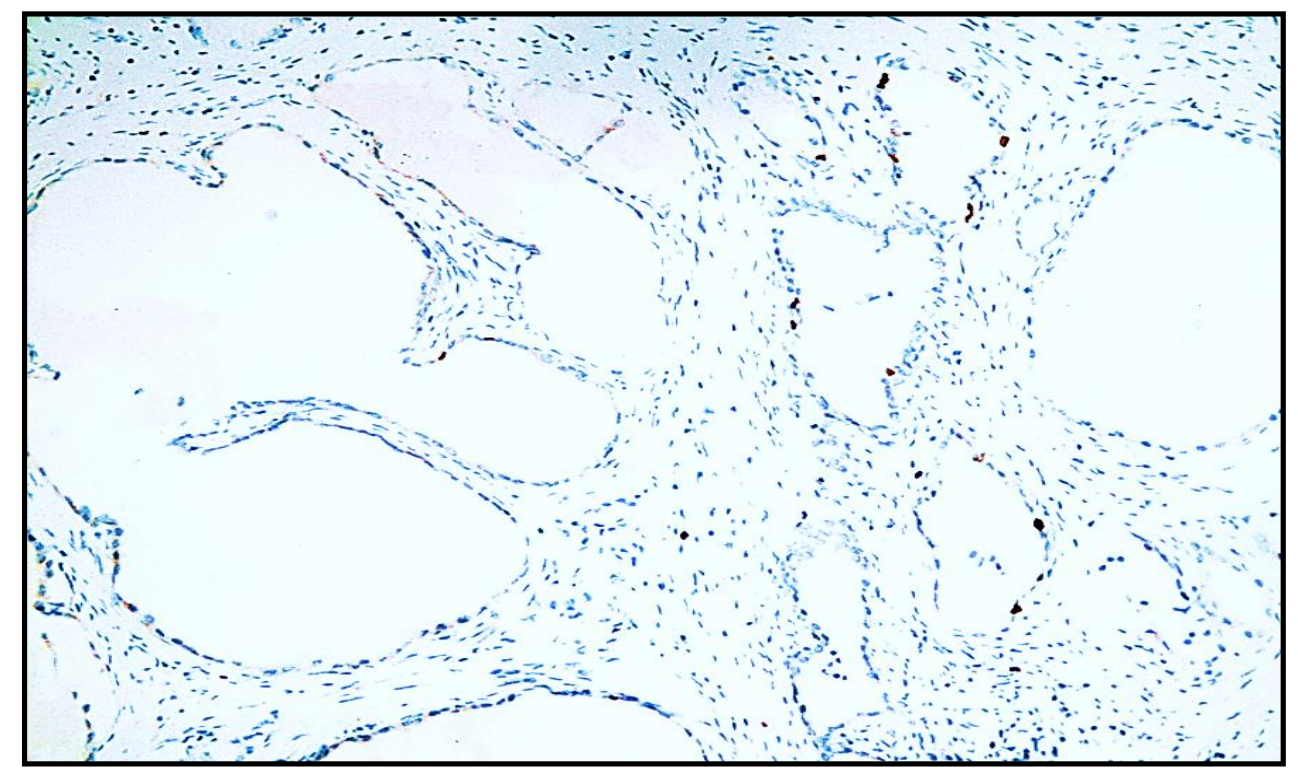

Figure (4):- Benign prostatic hyperplasia showing positive Ki 67 staining (Ki67x 200)

No significant relationship was found between the intensity of Ki-67 expression in prostate cancer cases and age of the patients $(\mathrm{P}=0.616)$, preoperative $\mathrm{PSA}(\mathrm{P}=0.199)$, but there is statistically significant positive correlation between Ki-67 positivity and increased Gleason's score $(\mathrm{P}<0.001)$ (Table 6).

Table (6):- Correlation between Ki-67 expression in prostatic carcinoma cases and age, PSA and Gleason score:-

\begin{tabular}{|ll|l|l|l|}
\hline & & Age & PSA & Gleason score \\
\hline Ki-67 & Pearson Correlation & -0.105 & 0.266 & 0.731 \\
P value & 0.616 & 0.199 & $<\mathbf{0 . 0 0 1}$ \\
\hline
\end{tabular}

A statistically significant positive correlation was observed between Cyclin D1 and Ki-67 expression in cancer group, PIN group and BPH group with $\mathrm{P}$ value $0.03,0.001$ and 0.04 respectively. 


\section{Discussion:-}

In the present study, twenty three of the prostate cancer cases $(92 \%)$ revealed positive nuclear staining for Cyclin D1. Nearly similar results were given by previous studies performed by Gupta et al. (2014) $)^{(\mathbf{1})}$, Ozbek et al (2000) $)^{(\mathbf{1 0})}$, Ueda et al.(2001) ${ }^{(\mathbf{1 3})}$ and Anis et al (2013) ${ }^{(\mathbf{1 5})}$ which reported positive nuclear Cyclin D1 expression ranging between $84.6 \%$ and $100 \%$, but the results were in contrast with Kallakury et al.(1997) ${ }^{(16)}$ Shiraishi et al. $\mathbf{( 1 9 9 8 )}^{\mathbf{( 1 7 )}}$ and Drobnjak et al. (2000) ${ }^{\mathbf{( 1 8 )}}$ which noticed positive nuclear Cyclin D1 expression in $22 \%, 30.3 \%$ and $11 \%$ of their studied cases respectively. Different techniques and methods used for detection of Cyclin D1 in this study might explain the discrepancy in results.

Regarding PIN, eight cases (80\%) showed positive Cyclin D1 expression. These results were close to previous studies by Gupta et al. (2014) ${ }^{(\mathbf{1})}$ and Anis et al. (2013) ${ }^{(\mathbf{1 5})}$ which reported positive nuclear Cyclin D1 expression in all studied PIN cases (100\%).

In BPH group, only 3 cases (20\%) showed positive nuclear Cyclin D1 expression. These results were in harmony with a previous work performed by Comstock et al. (2007) ${ }^{(\mathbf{1 4})}$ that reported positive nuclear Cyclin D1 expression in $13 \%$ of all cases. On the other hand, other works reported higher percentage of positively stained cases as it was $53.8 \%$ in the study done by Ueda et al.(2001) ${ }^{(\mathbf{1 3})}$ and $72.2 \%$ in Gupta et al. (2014) $)^{(\mathbf{1})}$.

No significant association or correlation was found between the intensity of Cyclin D1 expression in prostate cancer cases and other clinicopathological factors as age of the patient, preoperative PSA and Gleason scoring of cancer prostate cases. These results go with most of the previous researchers ${ }^{(13-18)}$, but they were in contrast with Ozbek et al.(2000) ${ }^{(\mathbf{1 0})}$ which observed a positive correlation between Gleason grade and staining intensity of Cyclin D1.

There was a statistically significant difference in Cyclin D1expression among BPH, PIN and carcinoma cases with the highest expression in carcinoma cases. These results were in concordance with a previous work by Gupta et al. (2014) ${ }^{(1)}$ which observed that cyclin D1 expression tends to increase in malignant prostate tissue.

Regarding the expression of Ki-67 in the studied cases, twenty (80\%) of the cancer prostate cases showed positive Ki- 67 expression. Our results are also close to those observed by other researchers; Muñoz et al (2003) ${ }^{(19)}$ and Verma et al $(\mathbf{2 0 1 5})^{(20)}$.

In PIN group; seven (70\%) cases showed positive Ki 67 staining. These results were different from those in the study done Sasor et al. (2000) $)^{(\mathbf{2 1})}$ in which only 3(30\%) out of 10 PIN cases exhibited Ki-67 positivity.

In the present study only one case $(6.7 \%)$ of BPH cases showed positive Ki 67 expression. These results are close to a previous study by Verma et al (2015) ${ }^{\mathbf{( 2 0 )}}$ which reported that Ki-67 was expressed in only one out of 10 (10\%) of BPH cases.

A statistically significant difference in expression of Ki-67 between BPH and prostate carcinoma was observed and this agrees with results of previous works ${ }^{(20,22,23)}$.

There was no significant association or correlation between the intensity of Ki-67 expression in prostate cancer cases and age of the patients, preoperative PSA, but there is statistically significant positive correlation between Ki-67 positivity and increased Gleason's score and this is in concordance with other studies ${ }^{(\mathbf{1 2}, 20,23,24)}$ which concluded that Ki-67 can be used as a prognostic factor for prostate cancer. But in contrast to a single work performed by Muñoz and his colleagues (2003) ${ }^{(\mathbf{1 9 )}}$ which detected no significant difference between the Gleason's score and Ki- 67 immunostaining.

In this study a statistically significant positive correlation was observed between Cyclin D1 and Ki-67 expression in cancer group, PIN group and BPH group, and this was similar to a previous study by Aaltomaa and his colleagues $(\mathbf{2 0 0 6})^{(25)}$. 


\section{Conclusion:-}

This study concluded that in prostatic carcinoma; Cyclin D1 immunohistochemical expression may help in diagnosis but it has no role as a prognostic factor. It was also concluded that Ki-67 can be applied along with other prostate cancer prognostic factors.

\section{References:-}

1. Gupta V., Garg M., Chaudhry M., Singh S., Sen R., Gill M., Sangwaiya A. Role of cyclin D1 immunoreactivity and Ag NOR staining in the evaluation of benign and malignant lesions of the prostate, Prostate Int. 2014;2:9096.

2. Dunn M., Kazer M. Prostate cancer overview. Semin OncolNurs. 2011; 27: 241-250.

3. Mokhtar N., Gouda I., Adel I. Malignant male genital tumor. In: Cancer pathology registry 2003-2004 and time trend analysis. Mokhtar N, Gouda I, Adel I (eds). El Sheraa. 2007; 94-96.

4. Cohen R., McNeal J., Baillie T. Patterns of differentiation and proliferation in intraductal carcinoma of the prostate: significance for cancer progression. Prostate. 2000; 43:11-9.

5. Diehl J. Cycling to cancer with cyclin D1. Cancer BiolTher. 2002; 1: 226-231.

6. Fleischmann A, Rocha C, Saxer-Sekulic N, Zlobec I, Sauter G, Thalmann G. High-level cytoplasmic cyclin D1 expression in lymph node metastases from prostate cancer independently predicts early biochemical failure and death in surgically treated patients. Histopathology. 2011; 58: 781- 789.

7. Berney DM., Gopalan A., Kudahetti S., et al. Ki-67 and outcome in clinically localized prostate cancer: analysis of conservatively treated prostate cancer patients from Trans-Atlantic Prostate Group study. Brit J Cancer. 2009; 1000: 888-893.

8. Nagao K., Yamamoto Y., Hara T., et al. Ki67 and BUBR1 may discriminate clinically insignificant prostate cancer in the PSA range < $4 \mathrm{ng} / \mathrm{ml}$. Jpn J ClinOnco. 2011; 41: 555-564.

9. Amin MB., Grignon DJ., Humphrey PA., Srigley JR. Contemporary application of Gleason grading in 18-gauge needle biopsy, transurethral resection and radical prostatectomy specimens. In:Gleason grading of prostate cancer: Amin MB, Grignon DJ, Humphrey PA and Srigley JR (eds). Philadelphia: Lippincott Williams \& Wilkins, 2004, 13-60 pp.

10. Ozbek E., Mirzak B., Ozbek M., Buyukberber S., Davarci M. Cyclin D1 protooncogen expression in prostate cancer. Turkish Journal of Cancer. 2000; 30: 15-21.

11. Raju Bina, Mehrotra Ravi, Øijordsbakken Gunnvor, Al-Sharabi Ali K., Vasstrand Endre N., Ibrahim Salah O. Expression of p53, Cyclin D1 and Ki-67 in Pre-malignant and Malignant Oral Lesions: Association with Clinicopathological Parameters. Anticancer Research. 2005; 25: 4699-4706.

12. Madani SH, Ameli S, Khazaei S, Kanani M, Izadi B. Frequency of Ki-67 (MIB-1) and P53 expressions among patients with prostate cancer. Indian J Pathol Microbiol. 2011; 54:688-91.

13. Ueda N., Yamashita M., Kuroda I., Takenaka I. Immunohistological evaluation of the expression of P27 and cyclin D1 in prostatic specimens. Nishinihon J Urol. 2001;63:246-9.

14. Comstock CE, Revelo MP, Buncher CR, Knudsen KE. Impact of differential cyclin D1 expression and localization in prostate cancer. Br J Cancer. 2007; 96:970-9.

15. Anis Ilia, Hosni Hala Naguib, Darweesh Mohammed F., Abd El Rahman Marwa. Immunohistochemical Expression of Cyclin D1 in Egyptian Patients with Prostatic Carcinoma. World Journal of Medical Sciences. 2013; 8 (4): 306-313.

16. Kallakury BV., Sheehan CE., Ambros RA., Fisher HA., Kaufman, RP., Ross JS. The prognostic significance of p34 and cyclin D1 protein expression in prostate adenocarcinoma. Cancer. 1997; 80: 753-763.

17. Shiraishi T., Watanabe M., Muneyuki T., Nakayama T., Moirita J., Ito H., Kotake T., Yatani R. A clinicopathological study of p53, p21 (WaF1/CIP1) and cyclin D1expression in human prostate cancer. UrologiaInternationalis. 1998; 61: 90-94.

18. Drobnjak M., Osman I., Scher HI., Fazzari M. Cardo CC. Overexpression of Cyclin D1 is associated with metastatic cancer to bone. Clinical Cancer Research. 2000; 6: 1891-1895.

19. Muñoz E., Gómez F., Paz J.I., Casado I., Silva J.M., Corcuera M.T., Alonso M. J. Ki-67 immunolabeling in pre-malignant lesions and carcinoma of the prostate. Histological correlation and prognostic evaluation, European Journal of Histochemistry. 2003; 2:123-128. 
20. Verma R., Gupta V., Singh J., Verma M., Gupta ., Gupta S., Sen R.v and Ralli M. Significance of p53 and ki-67 expression in prostate cancer. Urology Annals; Oct-Dec.2015, Vol. 7 Issue 4, p488.

21. Sasor A, Wagrowska-Danilewicz M, Danilewicz M. Ki-67 antigen and P53 protein expression in benign and malignant prostatic lesions. Immunohistochemical quantitative study. Pol J Pathol. 2000; 51:31-6.

22. Nikoleishvili D, Pertia A, Trsintsadze O, Gogokhia N, Managadze L, Chkhotua A. Expression of p27(Kip1), cyclin D3 and Ki67 in BPH, prostate cancer and hormone-treated prostate cancer cells. IntUrolNephrol. 2008; 40:953-9.

23. Rashed HE, Kateb MI, Ragab AA, Shaker SS. Evaluation of minimal prostatecancer in needle biopsy specimens using AMACR (p504s), p63 and Ki-67. Life Sci. 2012; 9:12-21.

24. Feneley MR, Young MP, Chinyama C, Kirby RS, Parkinson MC. Ki-67 expression in early prostate cancer and associated pathological lesions.JClin Pathol. 1996; 49:741-8.

25. Aaltomaa S., Kärjä V., Lipponen P., Isotalo T., Kankkunen J-P., Talja M., Mokka R. Expression of Ki-67, Cyclin D1 and Apoptosis Markers Correlated with Survival in Prostate Cancer Patients Treated by Radical Prostatectomy. Anticancer Research. 2006; 26: 4873-4878. 